\title{
EXPERIMENTAL COMPARISON BETWEEN FLAT AND CURVED SLIDING CONDITIONS FOR THE RESPONSE EVALUATION OF CURVED SURFACE SLIDER DEVICES
}

\author{
Alberto Pavese ${ }^{1}$, and Marco Furinghetti ${ }^{1,2}$ \\ ${ }^{1}$ University of Pavia \\ Via Ferrata 3, 27100 Pavia (Italy) \\ a.pavese@unipv.it \\ marco.furinghetti@unipv.it \\ ${ }^{2}$ EUCENTRE \\ Via Ferrata 1, 27100 Pavia (Italy) \\ marco.furinghetti@eucentre.it
}

\begin{abstract}
Curved Surface Slider devices have been widely used in last years for the protection of both building and structural systems. The spherical shape of the implemented sliding surfaces provide a certain recentering capability, which is generally combined to significant amount of energy dissipation, due to the frictional characteristics of the adopted sliding material. Since both behaviors act simultaneously during motion, experimental tests could return significantly high force values, especially if large bearings are considered. In some of those cases, the maximum force capacity of the testing equipment can be even overcome, and consequently experimental tests can not be performed.

The scope of the present work is to provide experimental evidence of the comparison between flat and curved sliding motions. Precisely, the outcomes of bi-directional tests performed on on full-scale Double Curved Surface Slider and Flat Slider devices have been analyzed. On the former typology the frictional and the recentering behaviors have been numerically decoupled, in order to compare the obtained results to the frictional response of the latter device. Results have shown a good agreement between the considered sliding motions, which seems to suggest that the experimental evaluation of flat sliding characteristics could be representative of curved sliding motions.
\end{abstract}

Keywords: Curved Surface Slider, Flat slider, base isolation, Experimental campaign, friction coefficient. 


\section{INTRODUCTION}

In last years the research about Curved Surface Slider devices has shown good performance in reducing the structural vulnerability of both buildings and bridge structures ([5] , [4] , [12] , [13] ). The overall response can be significantly mitigated, through the energy dissipation induced by the frictional behavior of the sliding motion, together with potentially low residual displacements, thanks to a properly designed recentering characteristics. In addition, the growing interest in investigating the experimental response of such devices have highlighted a number of important aspects to account for, such as some dependencies of the friction coefficient with respect to specific response parameters, in terms of sliding velocity, vertical load/contact pressure and cyclic effect ([6], [11] ). These dependencies have been detected, by analyzing the force response of both flat and curved sliding motions, and consequently a certain correlation between these different loading conditions is expected, regardless the applied loading conditions ([7], [9] , [2] ). From a theoretical perspective, the lateral force of a Curved slider can be computed as the summation of a recentering behavior, modeled as a linear spring with respect to displacements and a frictional response ([3] , [10] ). This relationship can be used to compare the frictional response of flat and curved sliding motions. If results between the aforementioned sliding conditions were actually comparable, testing possibility of curved devices would increase, since it would be possible to perform tests on the flat sliding material only, rather than the full curved isolator, and consequently much lower force demands could be achieved. In this work the outcomes of a wide experimental campaign on full-scale flat and curved sliders are analyzed, in terms of comparison of the resulting frictional properties. The considered dynamic tests have been performed at the Laboratory of EUCENTRE Foundation in Pavia, by adopting a special testing setup, in order to apply bidirectional motions.

\section{TESTED DEVICES}

In order to analyze the differences between flat and curved sliding motions, two individual typologies of sliding devices have been adopted. Precisely, full-scale isolators have been adopted, aiming at obtaining more realistic experimental results, as representative of the proper loading conditions of the devices ([6] ).

The former device consists of a Flat Slider, which returns the pure frictional behavior: it consists of a stainless steel flat sliding surface, which has been polished to mirror finish, by considering a roughness index $\mathrm{Ra} 0.2$, and a slider with a circular sliding pad. Such a device can be subjected to bidirectional displacements up to $250 \mathrm{~mm}$.

The latter isolator is a Double Curved Surface Slider, which represents one of the most common technology for isolation bearings. Two spherical sliding surfaces with the same curvature radius have been implemented, both polished to mirror finish in order to achieve the same roughness index of the Flat Slider: consequently, same frictional properties can be ensured; in addition, since the same radius of curvature has been designed for both the upper and the lower spherical surfaces, the global horizontal motion applied to the device can be considered as halved in two opposite sliding motions at the sliding interfaces. Within the equal sliding surfaces, a non-articulated slider is installed, which is made up of a unique steel block (material S355JR): such an element houses two circular sliding pads, having same diameter $(260 \mathrm{~mm})$ and same material composition, aiming at considering the same contact pressure and the same frictional properties at both the sliding interfaces. Both the radii of curvature of the spherical surfaces are $1600 \mathrm{~mm}$ each, with internal slider height of $120 \mathrm{~mm}$ : consequently, the equivalent radius of curvature of the device is approximately equal to $3080 \mathrm{~mm}$. 
In Figure 1 both the adopted isolation devices are shown.
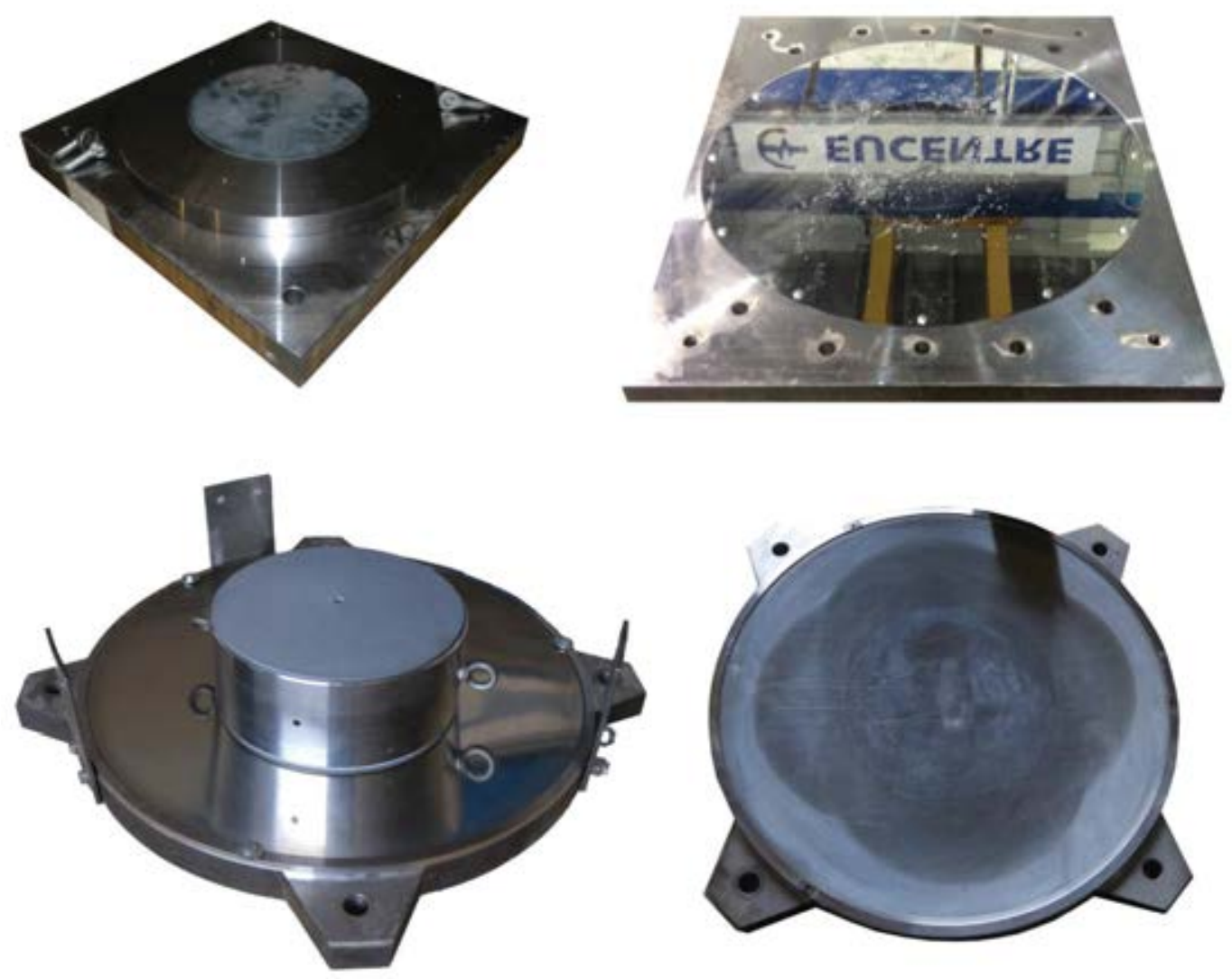

Figure 1: Full-scale Flat (top) \& Concave (bottom) Surface Slider devices (FS \& DCSS)

Isolation devices have been equipped with an innovative sliding material, which returns a controlled frictional response. Precisely, a pigmented graded PTFE filled with carbon fibres has been considered. The chemical formulation, such a material results in a much stiffer material in comparison to ordinary PTFE compositions. For both the typologies of isolation devices, three different sliding pad sizes have been considered, as shown in Figure 2.

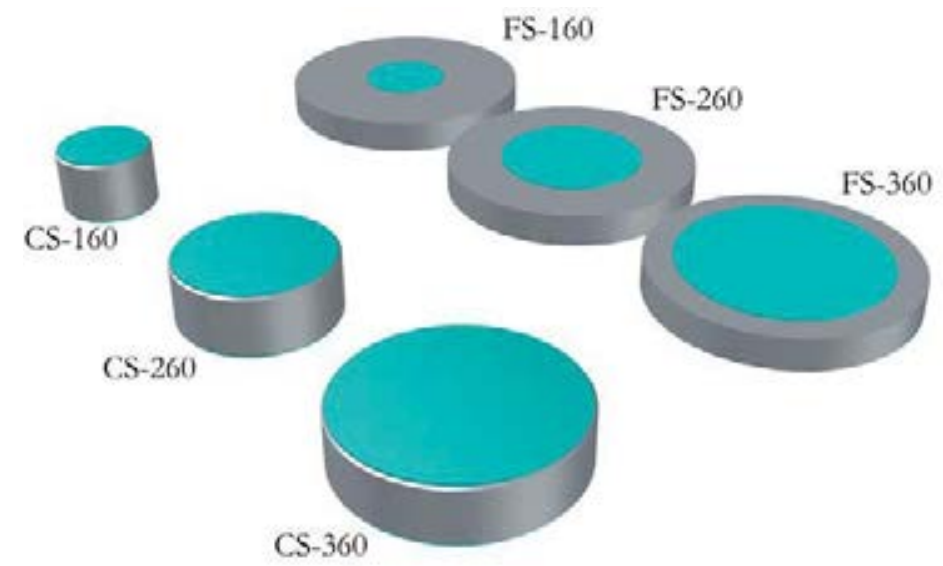

Figure 2: Internal sliders for Flat and Curved devices 
Thanks to the adopted sliders it is possible to analyze the comparison between flat and curved sliding conditions also for different sizes of the sliding pads, which may influence the distribution of the contact stresses at each interface and, consequently, the frictional response.

\section{BIDIRECTIONAL TESTING PROTOCOL}

The testing protocol has been defined, by considering bidirectional tests, according to the cloverleaf orbit, which is ruled by the European standard code UNI:EN15129:2009 for antiseismic devices ([1] ). Tests have been carried out through the Bearing Tester System at the Laboratory of the EUCENTRE Foundation in Pavia ([8] ), which provides a special equipment for the application of bidirectional horizontal motions to isolation devices. Specifically, the time series of horizontal displacement along both $\mathrm{x}$ and $\mathrm{y}$ directions for the cloverleaf orbits can be returned by the following expressions:

$$
\begin{aligned}
& x(t)=\frac{D_{\text {max }}}{\sqrt{2}} \cdot \sin (4 \pi f t) \cdot[\sin (2 \pi f t)+\cos (2 \pi f t)] \\
& y(t)=\frac{D_{\max }}{\sqrt{2}} \cdot \sin (4 \pi f t) \cdot[\sin (2 \pi f t)-\cos (2 \pi f t)]
\end{aligned}
$$

Such displacement time series have to be applied simultaneously to the device, which is consequently subjected to a bidirectional motion.

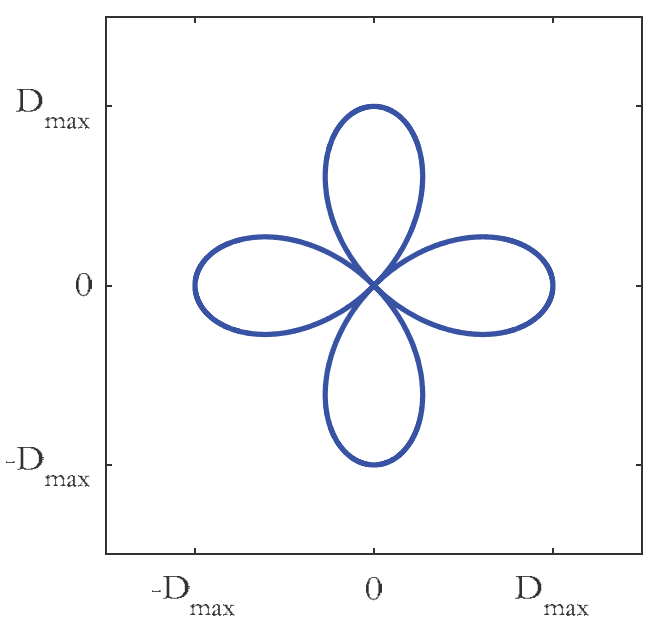

Figure 3: Cloverleaf bidirectional orbit

The aforementioned analytical definition of the displacement input leads to the following expression of the peak vectorial velocity of the test.

$$
\mathrm{V}_{\max }=4 \cdot \pi \cdot \mathrm{f} \cdot \mathrm{D}_{\text {max }}
$$

In order to better characterize the frictional properties of all the investigated devices, by ensuring a constant value of vectorial sliding velocity, input signals for bidirectional tests have been modified. Precisely, according to the aforementioned analytical definition of motion, the tangent velocity modulus varies between $50 \%$ and $100 \%$ of the peak value of the test, variations which may induce unexpected discrepancies in the overall frictional behavior of the considered device. Thus, a special resampling procedure has been applied to all tests, in order to obtain a constant modulus of the tangent velocity for the whole duration of motion. In Fig- 
ure 4 a comparison of the input signals for ordinary and CTV (Constant Tangent Velocity) orbits is provided.

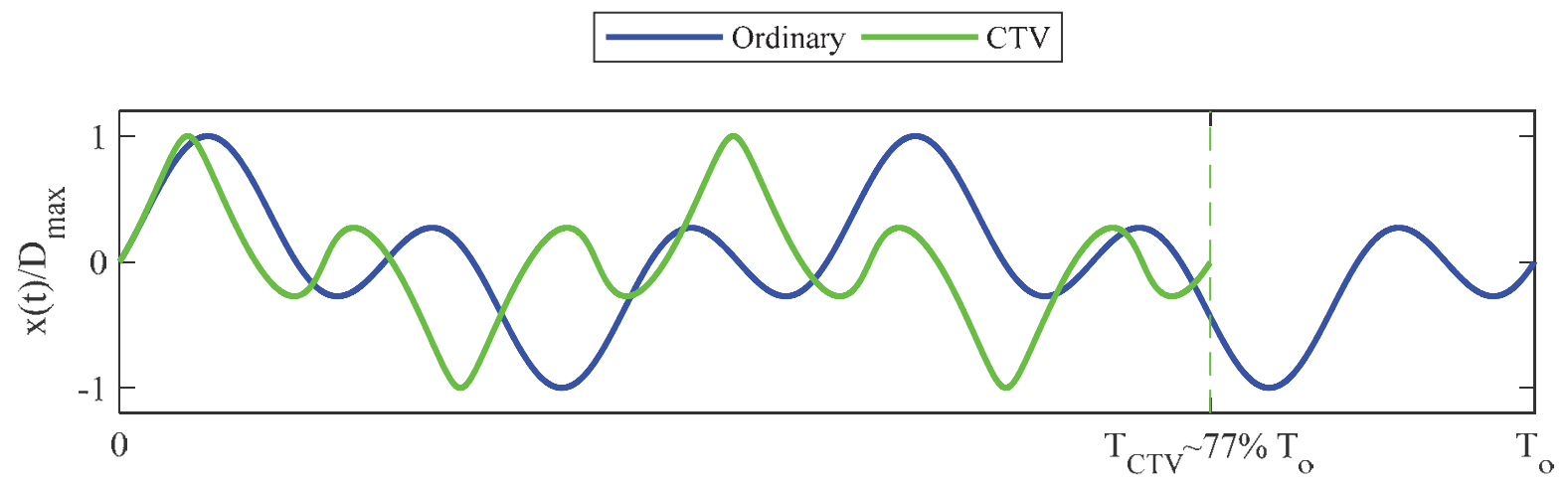

Time

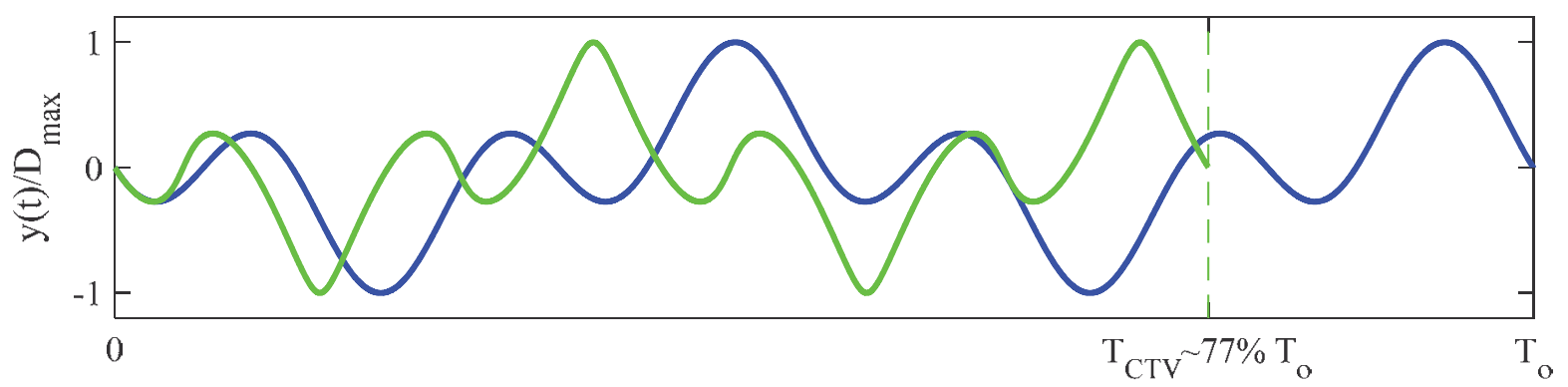

Time

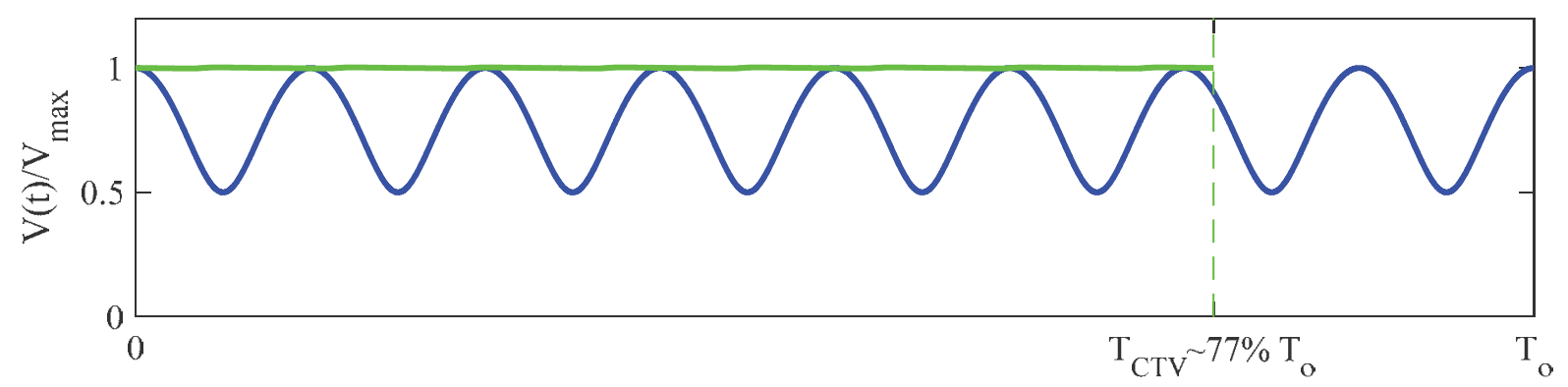

Time

Figure 4: Comparison between ordinary and CTV bidirectional orbits

The aim of the present research work is to directly compare the frictional response of both flat and curved sliding motions, by adopting different diameters of the implemented sliding pads, under bidirectional motions. In order to obtain to apply to the flat slider the effective motion of a single sliding interface of the curved isolator, the geometrical characteristics of the adopted spherical surfaces and the internal slider have been studied. Precisely, since the Curved Slider has the same radius of curvature for both the sliding surfaces, each sliding interface of the internal slider is subjected to $50 \%$ of the global displacement applied to the curved device; consequently, the velocity at the sliding interface is also halved. Hence, the testing protocol of the Flat Slider (FS) is obtained by halving peak values of both maximum displacement and velocity of the curved device.

In Table 1 the testing protocol for all the devices is summarized. 
Table 1. Bidirectional testing protocol for DCSS devices

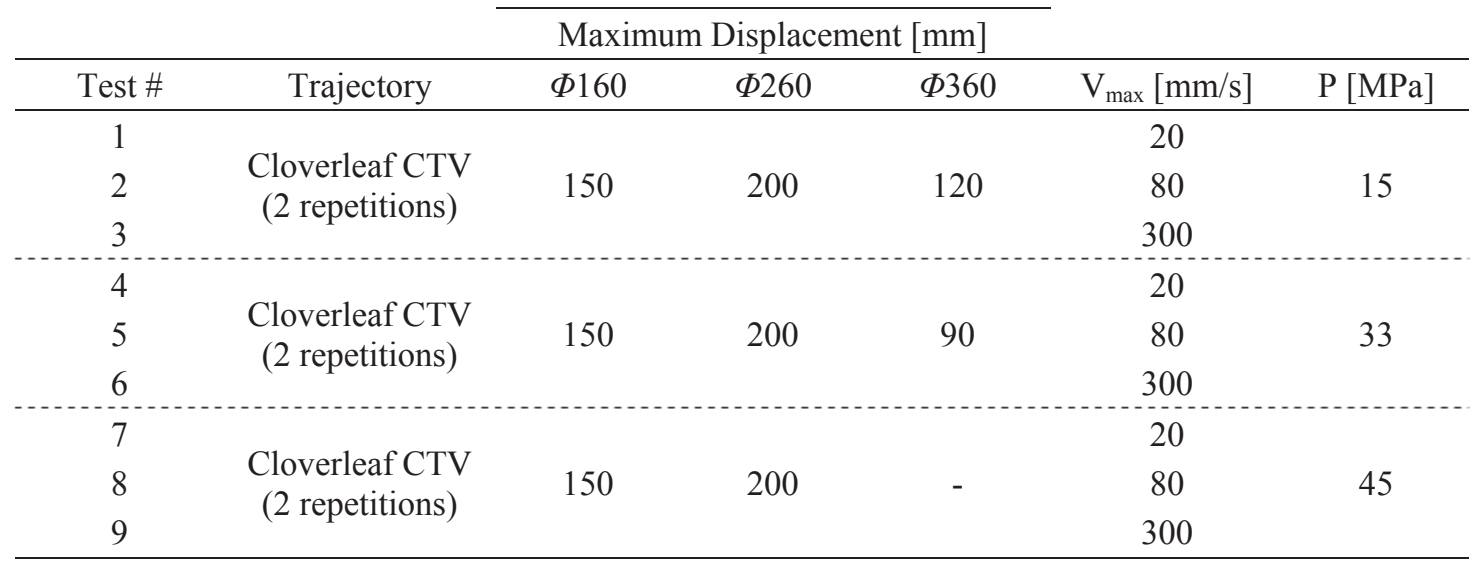

The vertical load of each test has been computed, by applying $15 \mathrm{MPa}, 33 \mathrm{MPa}$ and $45 \mathrm{MPa}$ contact pressures for each sliding pad diameter, and by considering two repetitions of bidirectional CTV cloverleaf orbit for all tests. It has to be noted that $45 \mathrm{MPa}$ pressure level has not been applied to the $360 \mathrm{~mm}$ sliding pad, due to the high resulting vertical load value.

\section{DEFINITION OF THE HYBRID FORCE RESPONSE}

The force response of a Concave Surface Slider (CSS) device is generally computed as the summation of the restoring force, provided by the stepwise projection of the applied vertical load along the tangent line to the sliding surface, and the curved frictional force, originated at the sliding interfaces. Thus, in order to compare the force response of the flat and the curved sliders, a hybrid force signal has been computed for both $\mathrm{x}$ and $\mathrm{y}$ directions, in terms of summation of an experimental flat frictional force, provided by tests carried out on the Flat Slider, and a numerical recenetring force, modeled as a linear spring with respect to displacements along $\mathrm{x}$ and $\mathrm{y}$ directions.

$$
F_{\text {Hybrid }}=F_{f-\text { Flat }}+F_{\text {rec-num }}=F_{f-\text { Flat }}+\frac{W}{R_{e q}}\left[\begin{array}{l}
x \\
y
\end{array}\right]
$$

The obtained hybrid forces can be directly compared to the force response of the curved device, and resulting frictional properties can be studied. To this aim, the same diameter of the implemented sliding pads for both the tested devices has been considered, and a proper scaled flat testing protocol has been defined, in order to apply same loading conditions to all the sliding interfaces.

\section{AVERAGE FRICTION COEFFICIENT PER CYCLE}

The cyclic behavior of the frictional response has been studied in terms of decay of the friction coefficient value, by applying an ad hoc analytical definition of the Average Friction Coefficient per cycle, comparable to the one ruled by the code UNI:EN15129:2009. In order 
to do so, a proper "bi-directional cycle" has to be defined for the adopted cloverleaf orbit, similarly to what is considered when unidirectional motions are appliyed. Under radial tests, sinusoidal waveforms are applied, and consequently the device within a single cycle has to reach the maximum and the minimum displacements respectively starting from the centred position (the maximum value of the displacement is reached twice along two opposite directions). Consequently, if two lobes of the cloverleaf trajectory are considered, the maximum vectorial displacement is reached twice along two different (and orthogonal) directions as well. Thus, a set of two lobes of the bidirectional cloverleaf orbit can be fairly considered as a bidirectional cycle, as shown in Figure 5.
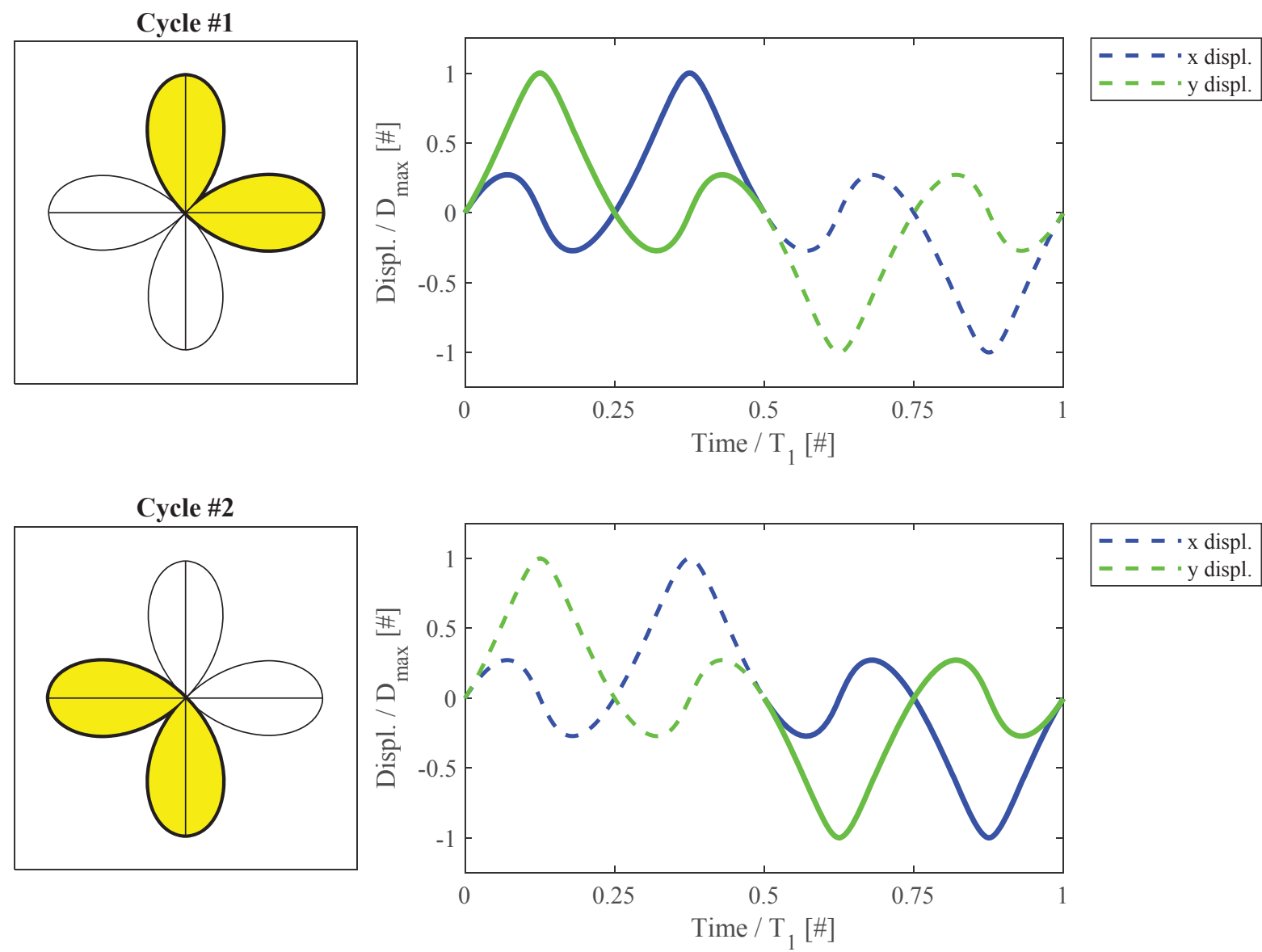

Figure 5: Definition of the bidirectional cycle

Thanks to this definition of the bidirectional cycle, for each test of the testing protocol of all devices four individual values of average friction coefficient per cycle can be computed, since the cloverleaf orbit has been repeated twice ( 8 lobes). The numerical value of the average friction coefficient per cycle can be computed, as a function of the Energy Dissipated per Cycle (EDC) and the product between the applied vertical load W and the total length of the bidirectional travelled path along two lobes $\left(4,844 D_{\max }\right)$.

$$
\mu_{E D C}=\frac{E D C}{4,844 D_{\max } W}
$$


The EDC value can be computed through the evaluation of the total work, in terms of integral of the scalar product between the force and the differential displacement vectors.

$$
E D C=\int \vec{F} \cdot d \vec{s}
$$

The computed average friction coefficient per cycle has to be referred to the dissipated energy value which occurs at half cycle point, which corresponds to the zero displacement point (undeformed configuration), between the achievement of the maximum displacement along the orthogonal directions (i.e. at the end of the first lobe of the bidirectional cycle).

\section{EXPERIMENTAL RESULTS}

In Figure 6, Figure 7 and Figure 8 results are shown for all the velocity levels, with a contact pressure equal to $15 \mathrm{MPa}, 33 \mathrm{MPa}$ and $45 \mathrm{MPa}$ respectively.
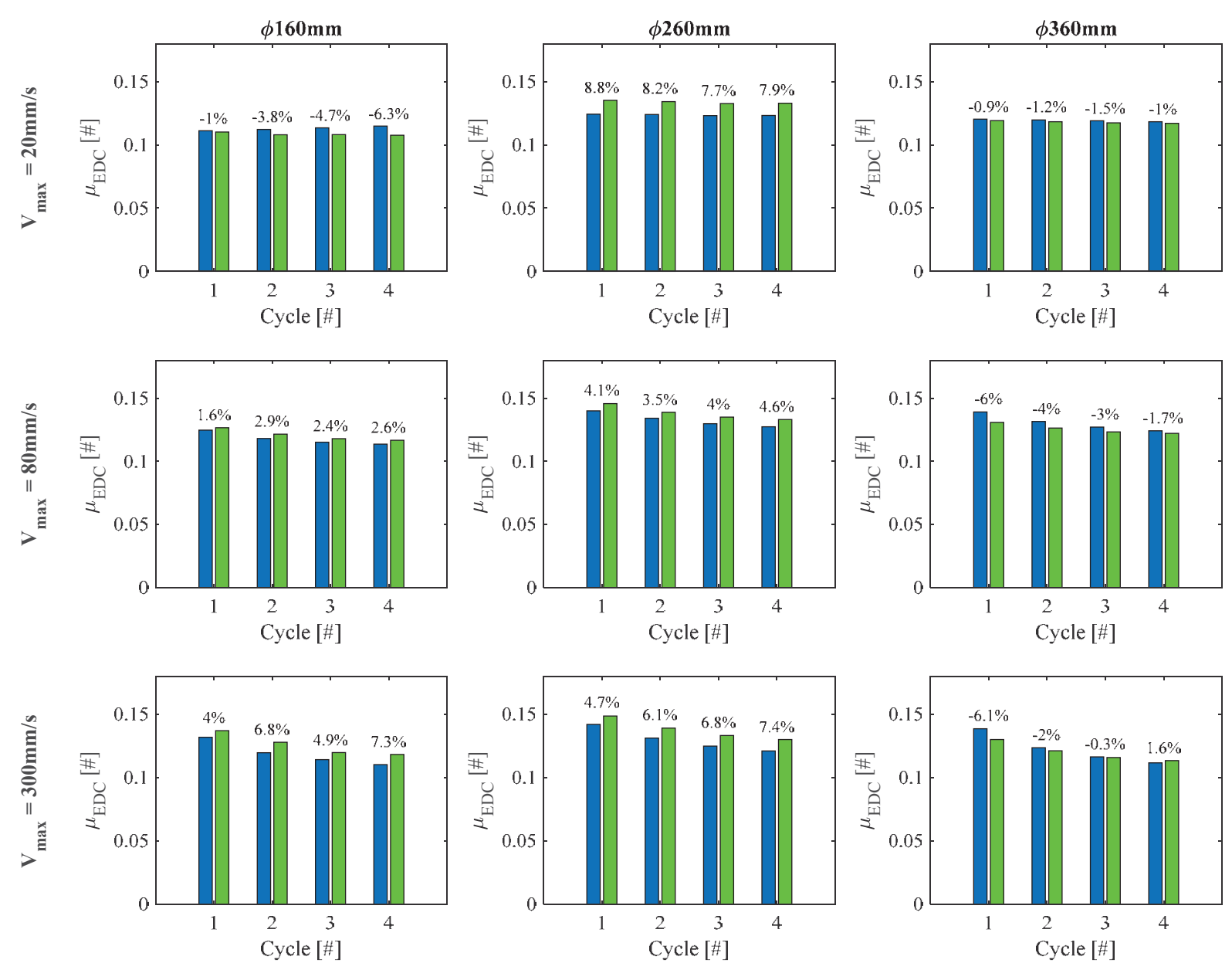

Figure 6: Average friction coefficient per cycle for Curved and Flat sliders, $\mathrm{P}=15 \mathrm{MPa}$. 

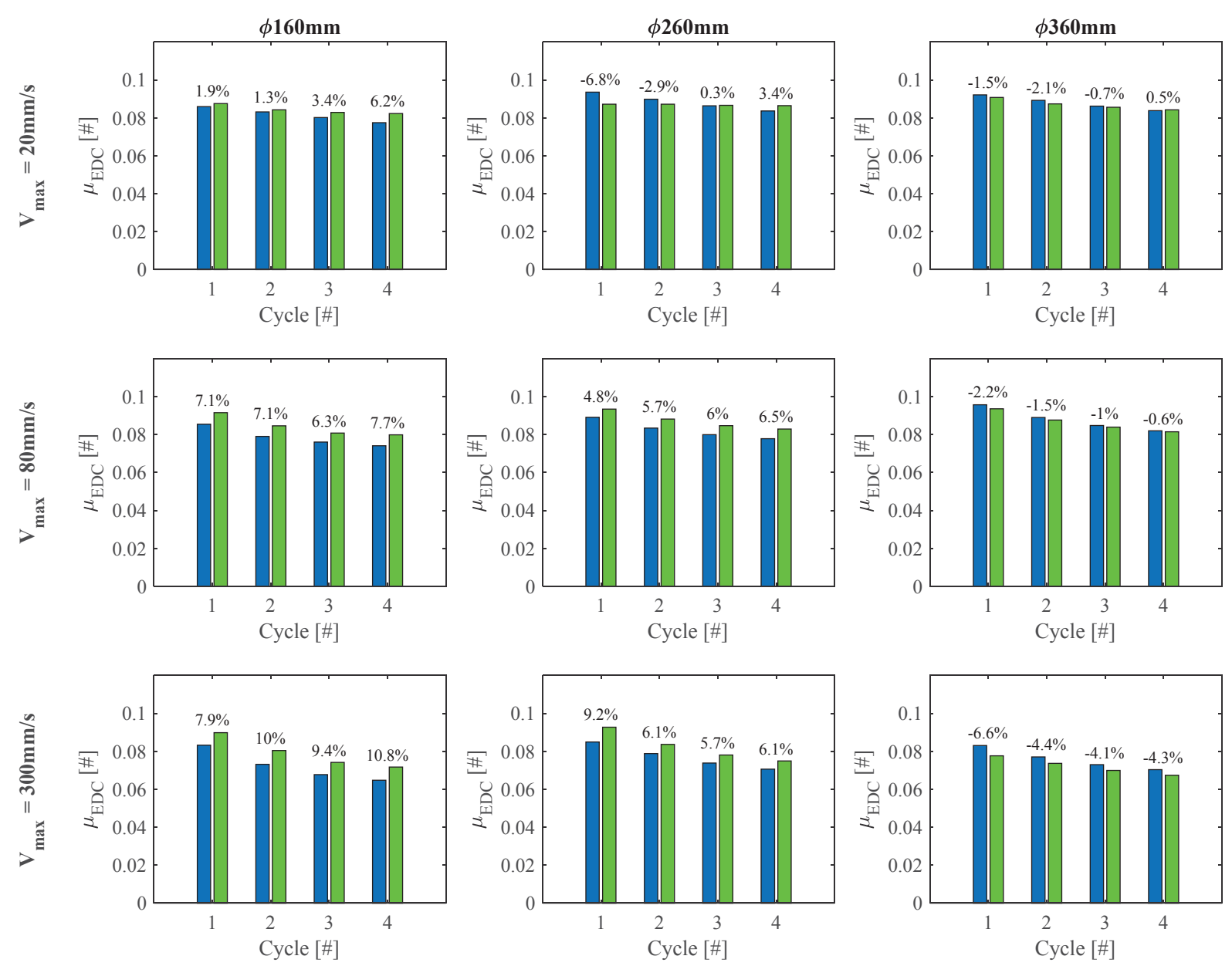

Figure 7: Average friction coefficient per cycle for Curved and Flat sliders, $\mathrm{P}=33 \mathrm{MPa}$.

Results are presented as values of average friction coefficient per each applied bidirectional cycle, which means four cycles for all the tests. More specifically, values have been computed, by considering the outcomes of tests performed on the Double Curved Surface Slider devices and the hybrid lateral response, computed as a combination of a numerical restoring force and the experimental flat frictional force. For the evaluation of the hybrid frictional characteristics, the displacement time series has been doubled, in order to consider the proper analogy with respect to the curved device. Results show a very good agreement between the frictional responses of the DCSS device and hybrid force related to the flat slider. Precisely, it can be assessed that for small-to-medium diameters of the sliding pad $(160 \mathrm{~mm}$ and $260 \mathrm{~mm})$ the hybrid friction coefficient is generally higher than the corresponding value related to the curved device, whereas for large diameters $(360 \mathrm{~mm})$ flat motions lead to lower friction coefficient values, in comparison to curved isolators, especially for high velocity. In all figures variation values are reported, in terms of variation of the friction coefficient returned by the hybrid response, with respect to the value related to the curved device. As can be noted, such variation values are less than $10 \%$, which highlight that the proposed method for frictional characterization provides a very good estimate of the real properties of the DCSS isolator. 

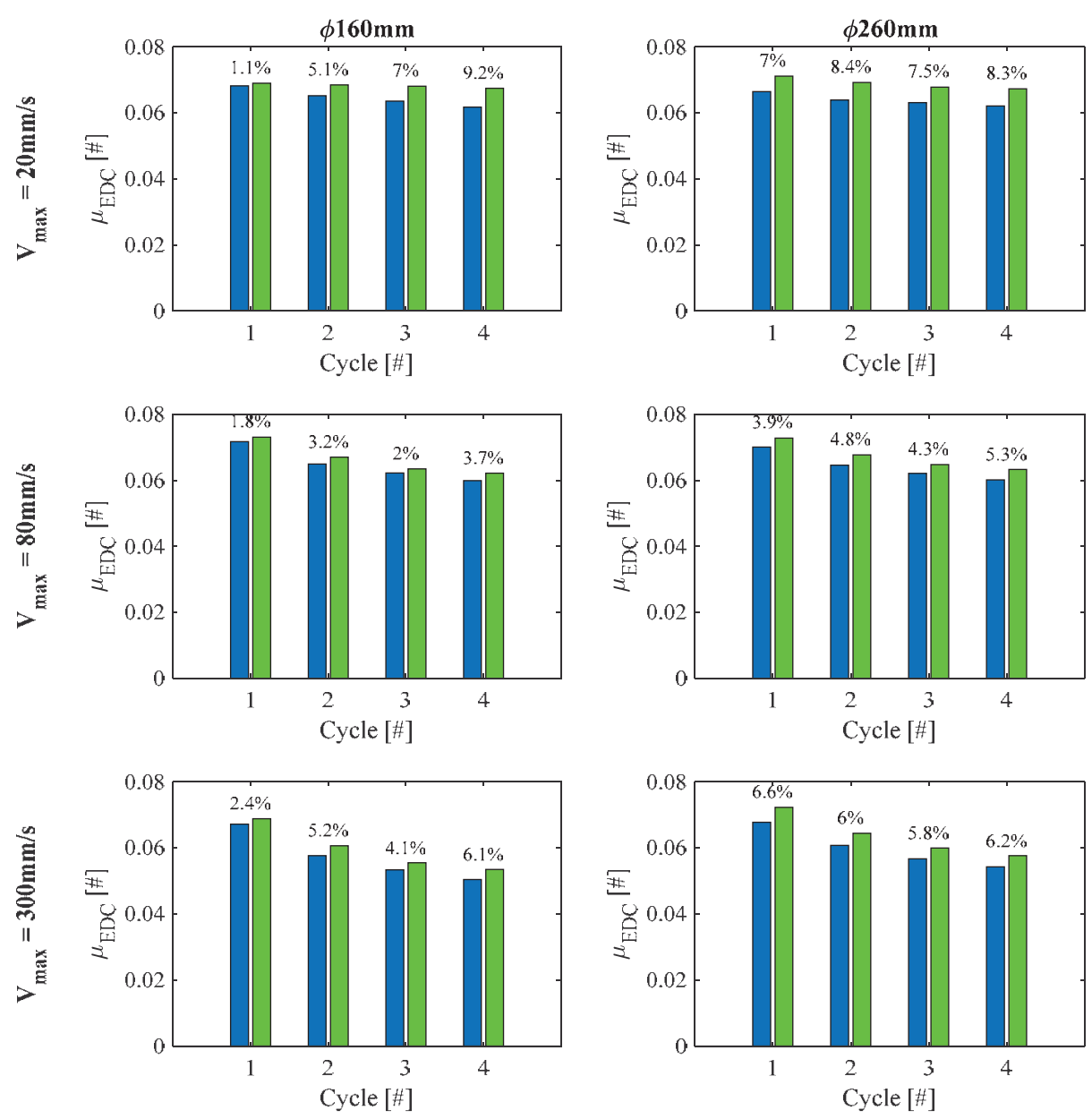

Figure 8: Average friction coefficient per cycle for Curved and Flat sliders, $\mathrm{P}=45 \mathrm{MPa}$.

Another aspect which can be noticed by analyzing graphical results is related to the frictional decay and the actual numerical values of the average friction coefficient per cycle. Precisely, it can be assessed that numerical values returned by the different sliding pad diameters re comparable, and approximately the same decay trend can be detected for all the velocity and contact pressure levels. This is a very important experimental evidence, which seems to prove that different diameters of the sliding pad actually lead to the same frictional properties, characterized by the same cyclic effect, if the proper motion is applied.

\section{CONCLUSIONS}

The presented experimental research provides the comparison between the frictional response returned by both flat and curved sliding motions. A large number of bidirectional dynamic tests have been performed at the Laboratory of EUCENTRE Foundation in Italy, by applying different loading conditions (i.e. contact pressure and sliding velocity) to ad hoc designed and realized full-scale devices, in order to provide results more comparable to applications commonly developed in the real practice. Precisely, Flat Sliders (FS) and Double Curved Surface Sliders (DCSS) have been adopted, by considering three individual sliding pad diameters. The testing program of the flat devices has been computed, by halving the peak displacement and velocity of the tests performed on the curved isolators, since both the 
spherical sliding surfaces have the same radius of curvature, and consequently the same sliding motion is originated at each sliding interface. Both the typologies of tested devices have been equipped with an innovative sliding material, which consists of a graded PTFE filled with carbon fibres. In order to carry out bidirectional tests, the cloverleaf orbit has been adopted, as ruled by the European standard code for anti-seismic devices UNI:EN15129:2009; in addition, displacement time series along both $\mathrm{x}$ and $\mathrm{y}$ directions have been resampled, in order to obtain a constant tangent velocity modulus for the whole duration of all tests. Average friction coefficient per cycle values have been computed, according to a proper definition of bidirectional cycle for the adopted orbit, and Flat and Curved frictional properties have been compared, by means of a hybrid response: such a force response can be computed as the summation between the experimental frictional force of the Flat Slider device and a numerical recentering force, modeled as a linear spring with respect to displacements (displacement time series have been doubled, in order to consider the actual motion of the curved devices). Results have been analyzed, in terms of average friction coefficient per each bidirectional cycle (four full cycles for each tests).

According to the presented results, it can be assessed that:

- The proposed hybrid response, computed as a function of the flat slider outcomes, leads to a very good estimate of the frictional properties of the corresponding curved device;

- The variation between the hybrid and the curved responses is bounded between $\pm 10 \%$, and in most of cases the average friction coefficient per cycle achieves higher values, when the hybrid force is adopted, especially for small-to-medium diameters of the sliding pads, and for high velocities;

- Different sliding pad diameters seems to lead to approximately same frictional properties, from both numerical values and cyclic effects (decay trend of the friction coefficient) points of view.

The obtained results look extremely promising, since curved sliding motions for the considered devices can be actually represented by equivalent flat responses, with small variations of the correspondent frictional behavior. The importance of such a conclusion can be noticed from both an economic and a technical standpoints: if the recentering contribution can be actually numerically modeled and the frictional response can be tested by considering an equivalent flat device, the behavior of Curved Surface Slider (CSS) devices can be easily captured by the definition of the hybrid response, and consequently large devices can be fully characterized, even though large force demands are experienced. Consequently, if the testing equipment is able to perform tests on the flat slider, the response of the curved isolator can still be obtained; nonetheless, if the capacity of the testing equipment is not enough to perform tests on the full-scale flat slider, a scaled sliding pad can be considered. Such results may need further experimental investigations, by analyzing different diameters of the sliders, rather than other sliding materials, commonly adopted in real practice applications.

\section{ACKNOWLEDGMENTS}

Part of the current work has been carried out under the financial support Italian Civil Protection, within the framework of the Executive Project DPC-EUCENTRE 2014-2016 (Project S2.0 - Seismic isolation and supplemental damping systems: evaluation of the seismic response of devices and structures) and ReLUIS project 2019-2021 (WP15, Task 15.1). 


\section{REFERENCES}

[1] CEN (2009): Comité Européen de Normalisation TC 340, European Code UNI EN 15129:2009 Anti-seismic devices, European Committee for Standardization. Brussels, Belgium.

[2] Dolce M, Cardone D, Croatto F (2005). Frictional behavior of steel-PTFE interfaces for seismic isolation. Bulletin of Earthquake Engineering, 3, pp. 75-99.

[3] Fenz D, Constantinou MC (2006). Behaviour of the double concave friction pendulum bearing. Earthquake Engineering And Structural Dynamics, 35, pp. 1403-1424.

[4] Furinghetti M., Lanese I., Pavese A. (2020) Experimental assessment of the seismic response of a base isolated building through hybrid simulation technique, Frontiers in Built Environment, DOI: 10.3389/fbuil.2020.00033.

[5] Furinghetti M., Pavese A. (2020) Definition of a Simplified Design Procedure of Seismic Isolation Systems for Bridges, Structural Engineering International, DOI: 10.1080/10168664.2020.1775535.

[6] Furinghetti M., Pavese A., Quaglini V., Dubini P. (2019) Experimental Investigation Of The Cyclic Response Of Double Curved Surface Sliders Subjected To Radial And Bidirectional Sliding Motions, Soil Dynamics and Earthquake Engineering, DOI: 10.1016/j.soildyn.2018.11.020.

[7] Lomiento G, Bonessio N, Benzoni G (2013): Concave sliding isolator's performance under multi-directional excitation. Ingegneria Sismica, 30 (3), 17-32.

[8] Peloso S, Pavese A, Casarotti C (2012): EUCENTRE TREES lab: Laboratory for training and research in earthquake engineering and seismology. Geotechnical, Geological and Earthquake Engineering, 20, 65-81.

[9] Quaglini V, Bocciarelli M, Gandelli E, Dubini P (2014): Numerical Assessment of Frictional Heating in Sliding Bearings for Seismic Isolation. Journal of Earthquake Engineering, 18 (8), 1198-1216.

[10] Quaglini V, Gandelli E, Dubini P (2017). Experimental investigation of the re-centring capability of curved surface sliders. Structural Control and Health Monitoring, 24, Issue 2, DOI: $10.1002 /$ stc. 1870 .

[11] Quaglini V., Dubini P., Furinghetti M., Pavese A. (2019). Assessment of Scale Effects in the Experimental Evaluation of the Coefficient of Friction of Sliding Isolators. Journal of Earthquake Engineering, DOI: 10.1080/13632469.2019.1687054.

[12] Tsiavos A, Schlatter D, Markic T, Stojadinovic B. (2017). Experimental and analytical investigation of the inelastic behavior of structures isolated using friction pendulum bearings. Procedia engineering, 199:465-70. 
[13] Tsiavos A, Schlatter D, Markic T, Stojadinovic B. (2021) Shaking table investigation of inelastic deformation demand for a structure isolated using friction-pendulum sliding bearings. Structures, 31, 1041-1052. 